\title{
\%
}

María del Mar García Galán*

Beatriz Román Suero*

Antonio Chamorro Mera*

\section{IMAGEN DE ESPAÑA COMO PRODUCTOR DE ACEITE DE OLIVA: UN ANÁLISIS ENTRE IMPORTADORES}

El aceite de oliva forma parte de la dieta mediterránea y tiene una imagen mundial de alimento de calidad y saludable. En las últimas dos décadas se han observado cambios significativos en la estructura de su consumo mundial, con un aumento significativo en quince años de las exportaciones mundiales. Así, pues, potenciar la imagen del país de origen del aceite se convierte en un elemento clave para su comercialización. Este estudio analiza la imagen que importadores de aceite de oliva de Alemania, México y Estados Unidos tienen de España como productor de aceite de oliva y su posible efecto en la preferencia por el aceite español frente a otros de origen italiano y griego. Los importadores consultados han mostrado tener una buena imagen de España como productor de aceite de oliva en los tres mercados internacionales analizados. En términos de imagen, el aceite italiano es el principal competidor del aceite español, muy por encima del griego, especialmente en el mercado alemán. Los puntos débiles de la imagen de España están relacionados con aspectos comerciales y de marketing (variedad de la oferta y atractivo de los diseños), precisamente los puntos fuertes de la oferta italiana. El sector tiene que centrar sus esfuerzos en mejorar en estos aspectos.

Palabras clave: origen, imagen de país, competidores.

Clasificación JEL: M31, Q13.

\section{Introducción}

El aceite de oliva solo representa el $2 \%$, aproximadamente, de la producción mundial de aceites y grasas, muy por debajo de las cifras del aceite de soja (25\%) y del aceite de girasol

\footnotetext{
* Departamento de Dirección de Empresas y Sociología, Facultad de Ciencias Económicas y Empresariales, Universidad de Extremadura. Versión de febrero de 2019.

DOI: https://doi.org/10.32796/bice.2019.3109.6779
}

(9\%), y los países productores son también los mayores consumidores. Sin embargo, en las últimas dos décadas se están observando cambios significativos en la estructura del consumo mundial y de las exportaciones de este producto, extendiéndose fuera de los tradicionales países productores.

Utilizando las estadísticas publicadas por el Consejo Oleícola Internacional (COI, 2019), las exportaciones han crecido significativamente en los últimos quince años. Así, la media de $\triangle$ 
las exportaciones en las últimas cinco campañas ${ }^{1}$ (de la 2013-2014 a la 2017-2018) fue de 846.100 toneladas, lo que supone un $17,4 \%$ más que en el quinquenio anterior (720.600 t) y un $35,6 \%$ más que dos quinquenios antes (623.800 t). El aumento del consumo en países no productores está compensando el estancamiento o la reducción del consumo interno en los tradicionales países productores.

En gran medida se puede considerar que estos cambios vienen favorecidos por la ampliación del segmento de consumidores LOHAS (Lifestyle Of Health And Sustainability), para quienes la salud es un criterio relevante en las decisiones de compra y consumo de alimentos. El aceite de oliva forma parte de la dieta mediterránea, declarada por Unesco como patrimonio intangible de la humanidad y tiene una imagen mundial de alimento de calidad y saludable que ha facilitado su penetración en mercados ajenos a los países productores.

En este contexto de crecimiento de las exportaciones a países no productores, tanto empresarios como administraciones públicas responsables de la política comercial exterior son conscientes de que potenciar la imagen del país de origen del aceite se convierte en un elemento clave para su comercialización. El origen del producto se convierte en muchos casos en un criterio relevante en las decisiones de compra del cliente, dando lugar al denominado «efecto país de origen». Bilkey y Nes (1982) lo definieron como «el grado en que la procedencia de un producto afecta al comportamiento del consumidor en los procesos de compra». En estos mercados exteriores la competencia se produce, en gran medida, entre países productores, más que entre marcas privadas. Además,

Se ha calculado la media de exportaciones por quinquenios para evitar el efecto de las fluctuaciones anuales del volumen de producción. al tratarse de segmentos de consumidores minoritarios, las acciones de marketing deben ir más enfocadas a los importadores y distribuidores, para conseguir un hueco en sus lineales de venta, que al propio consumidor final.

Teniendo presente lo anterior, la finalidad del presente estudio es analizar la imagen que los importadores de aceite de oliva tienen de España como productor de aceite de oliva y su posible efecto en la preferencia por el aceite español frente a otros de origen italiano y griego, los otros dos grandes productores mundiales de aceite de oliva. Los resultados obtenidos permiten extraer conclusiones útiles para las empresas agroalimentarias españolas del sector, así como para la política pública de promoción exterior, pues identifican las principales fortalezas y debilidades de la imagen internacional del aceite de oliva español.

La novedad de este estudio radica en que otros previos han analizado los criterios de compra y las preferencias de los consumidores finales de aceite de oliva en distintos mercados nacionales (principalmente países productores), pero no se han encontrado trabajos que se centren en el estudio de los importadores, ni que analicen expresamente la imagen del país productor.

\section{El origen como criterio de compra de aceite de oliva}

Las reacciones de los compradores ante el origen se han estudiado desde hace décadas en el ámbito del marketing agroalimentario, habiéndose demostrado que este tiene un peso importante en la decisión de compra de determinadas categorías de producto, ya que aporta confianza a los consumidores, disminuyendo el riesgo percibido. 
Gran parte de las investigaciones sobre las preferencias de compra de aceite de oliva por parte de los consumidores han incluido el origen como uno de los criterios de compra relevantes, tanto en los estudios realizados en países productores (Fotopoulos y Krystallis, 2001; Van der Lans et al., 2001; Scarpa y Del Guidice, 2004; Krystallis y Ness, 2005; Bernabéu et al., 2009; Mtimet et al., 2013; Erraach et al., 2014; Yangui et al., 2014) como en países no productores (García et al., 2002; Mtimet et al., 2008; Menapace et al., 2011). El efecto país de origen se ha analizado comparando su influencia en las decisiones de compra respecto a la de otros atributos como el precio, determinadas características organolépticas (color y sabor), el packaging, el tipo de producción o las certificaciones de calidad (principalmente las denominaciones de origen).

Se puede concluir que, en la mayoría de los mercados, el origen y el precio son los principales criterios en la decisión de compra de aceite de oliva. En este sentido, la relación origen-precio es contradictoria, pues en la literatura previa sobre los criterios de compra de aceite de oliva se han encontrado algunos estudios que obtuvieron que el origen era más importante que el precio (Fotopoulos y Krystallis, 2001; Krystallis y Ness, 2005; Mtimet et al., 2008; Bernabéu et al., 2009), pero también existen otros donde se obtiene el orden inverso, con el precio como principal determinante de la compra (Van der Lans et al., 2001; Scarpa y Del Giudice, 2004; Chan-Halbrendt et al., 2010; Menapace et al., 2011; Mtimet et al., 2013; Dekhili et al., 2011; Erraach et al., 2014).

Los estudios previos también ponen de manifiesto que el origen suele tener mayor importancia relativa que la apariencia y el packaging empleado para su comercialización (Van der Lans et al., 2001; Scarpa y Del Giudice, 2004; Krystallis y Ness, 2005; Menapace et al., 2011;
Erraach et al., 2014). Lo mismo sucede con el sistema de producción (convencional vs ecológico). Aunque los consumidores dan preferencia a los aceites de producción ecológica frente a los convencionales, los estudios previos concluyen que el sistema de producción es un aspecto menos relevante que el origen en la formación de la estructura de preferencias de los consumidores (Krystallis y Ness, 2005; Bernabéu et al., 2009; Menapace et al., 2011; Yangui et al., 2014).

Los resultados de la importancia del origen en comparación con las características organolépticas son menos concluyentes. El color del aceite resultó ser más relevante que el origen en la formación de las preferencias de los consumidores en los estudios de Van der Lans et al. (2001), Mtimet et al. (2008 y 2013), pero menos importante para los consumidores españoles analizados por Erraach et al. (2014). Con respecto al sabor, Chan-Haldbrendt et al. (2010), Menapace et al. (2011) y Panico et al. (2014) obtuvieron que su importancia relativa era menor a la del origen, mientras que el resultado fue el contrario en el estudio realizado en Túnez por Mtimet et al. (2013).

Los estudios realizados en países no productores han analizado el efecto país de origen en relación con otros atributos del aceite, así como la preferencia por el aceite de varios países productores. Los resultados de estos estudios son similares a los obtenidos en los países productores con relación a la alta importancia relativa del origen en la estructura de preferencias de los compradores. La excepción a este resultado es el estudio de García et al. (2002) entre consumidores ingleses, quienes pusieron de manifiesto que estos consumidores valoraban especialmente el precio, el tamaño y el formato en el proceso de compra, sin destacar el origen de procedencia. 
Respecto a la preferencia por distintos orígenes nacionales del aceite de oliva, tanto el estudio de Mtimet et al. (2008) como el de Menapace et al. (2011) reflejaron la mayor preferencia de los consumidores por el aceite italiano. Así, el estudio de Mtimet et al. (2008) entre consumidores japoneses obtuvo que el origen italiano resultó ser el más valorado, y después el español y el tunecino. Los resultados del estudio de Menapace et al. (2011) entre consumidores canadienses mostraron que el origen italiano era preferido al español e implicaba la disponibilidad a pagar un precio premium. Sin embargo, estadísticamente existía indiferencia entre los aceites griegos y españoles.

Finalmente, hay que indicar que los resultados generales de estos estudios sobre las preferencias de los consumidores de aceite de oliva son similares a los obtenidos en otros estudios que han analizado el efecto del origen en las valoraciones sensoriales y en la percepción de calidad del aceite de oliva (Ward et al., 2003; Caporale et al., 2005; Espejel y Fandos, 2008; Dekhili y D'Hauteville, 2009). Se puede decir que el origen del aceite es empleado por los consumidores como un indicador de la calidad percibida y tiene una influencia positiva en las expectativas sensoriales.

\section{La imagen del país de origen}

El mayor o menor efecto que el país de origen tiene en las intenciones o preferencias de compra de los clientes viene influido por determinadas variables explicativas 0 antecesoras; entre ellas la literatura previa suele identificar el etnocentrismo del consumidor, la animosidad hacia un determinado origen, la familiaridad con respecto al producto y al país, la imagen del país de origen, así como determinados factores sociodemográficos del consumidor.

Aunque se trata de un concepto muy recurrente en los estudios sobre el «efecto país de origen", la imagen del país de origen es un concepto ambiguo y multidimensional que ha sido definido y medido de forma muy heterogénea (Roth y Diamantopoulos, 2009). En algunos casos se identifica exclusivamente con la imagen país (o marca-país) para referirse a la imagen general que se tiene de un país a través de sus personas, su cultura, su economía y sus productos, instituciones y empresas. Molina (2004) definió la marca-país como «un conjunto de percepciones, asociaciones, recuerdos y prejuicios que la gente procesa en su mente y cuya síntesis es una imagen mental del país como producto".

En otras ocasiones la imagen país de origen se identifica exclusivamente con la imagen del país como fabricante de productos (por ejemplo, Askegaard y Ger, 1998; Papadopoulos y Heslop, 2003); es decir, se centra solo en la dimensión producto, por lo que suele referirse a ella como imagen producto-país o imagen made-in. Han (1989) lo define como la percepción general que los consumidores tienen de la calidad de los productos hechos en un determinado país, mientras que Roth y Romeo (1992) añaden que viene determinada por las valoraciones que el cliente hace sobre las fortalezas y debilidades de la producción y comercialización del país en cuestión.

La imagen producto-país puede referirse a una imagen global donde no se especifica ninguna categoría de producto, o puede centrarse exclusivamente en un tipo de producto concreto (en el caso de este artículo es el aceite de oliva). En este caso, la imagen país de origen evalúa la imagen del producto y del país como productor de esa categoría de producto (imagen producto). 
Se han desarrollado diversas propuestas de escalas para medir la imagen de los productos de un país. Por ejemplo, Roth y Romeo (1992) propusieron cuatro dimensiones para estudiar esta imagen: capacidad de innovación, diseño de los productos, prestigio y mano de obra. De forma similar, Wang et al. (2012) agruparon los ítems que pueden medir la imagen del país de origen en cinco factores: calidad, presencia global de marca, mano de obra, confianza y diseño. Como estos factores no se adaptan a todo tipo de productos, Van Ittersum et al. (2003) emplearon una escala para medir esta imagen adaptada al ámbito agroalimentario, considerando dos dimensiones generales: el factor humano y el factor ambiental, que refleja las condiciones naturales y climáticas de un lugar para producir el producto.

\section{Diseño de la investigación}

Como se ha indicado, la finalidad principal de este trabajo es analizar la imagen que los importadores tienen de España como país productor de aceite de oliva en comparación con los otros dos grandes productores internacionales: Italia y Grecia. Como población de estudio se han seleccionado los importadores que colaboraron en el proyecto «Alimentos de Extremadura - Foods from Spain» en 2014. Se trata de importadores que han trabajado o solicitado información para trabajar con productores de esta región española, cuarta en volumen de producción de aceite de oliva. De ellos se han seleccionado tres países muy diferentes geográfica y culturalmente: Estados Unidos, Alemania y México (Tabla 1).

Alemania es uno de los mercados más importantes fuera de los países productores. El aceite de oliva es el tercer aceite comestible
TABLA 1

FICHA TÉCNICA DE LA INVESTIGACIÓN

\begin{tabular}{|l|l|}
\hline Universo & $\begin{array}{l}\text { Importadores de aceite de oliva } \\
\text { Base de datos de colaboradores } \\
\text { del Proyecto Alimentos de } \\
\text { Extremadura en Alemania, } \\
\text { Estados Unidos y México } \\
\text { Tamaño de la muestra }\end{array}$ \\
Ámbito geográfico & $\begin{array}{l}\text { Alemania, Estados Unidos y } \\
\text { México } \\
\text { Estudio censal } \\
\text { Muestreo }\end{array}$ \\
Tipo de encuesta & $\begin{array}{l}\text { Online } \\
\text { De enero a mayo de } 2015\end{array}$ \\
Trabajo de campo & Fuente: elaboración propia. \\
\hline
\end{tabular}

más consumido en Alemania, con una cuota de mercado aproximada del $18 \%$, por detrás del aceite de colza y del aceite de girasol. Los alemanes consumieron 0,8 kilos de aceite de oliva per cápita en 2014, cifra que duplica el consumo de 2011 (COI, 2017). Según los datos de Eurostat, aproximadamente el $72 \%$ de las importaciones son de origen italiano, mientras que el $15 \%$ son de aceite español y el $9 \%$ de aceite griego. En valores absolutos, Alemania ocupa el puesto 14 en el ranking de destinos de las exportaciones de aceite de oliva español.

Según las estadísticas del COI, Estados Unidos es el mayor importador mundial de aceite de oliva fuera de la zona de producción tradicional. El consumo per cápita de aceite de oliva en Estados Unidos ha aumentado en las últimas décadas hasta alcanzar los 1,02 litros/ habitante según el United States Department of Agriculture (2015). El origen de sus importaciones está encabezado por Italia, con el $44 \%$ del total, seguido de España, con el 40\%, y Túnez, con casi un $16 \%$. Hace dos décadas, el porcentaje de las importaciones procedentes de España era de 30, lo que significa que la cuota de mercado de aceite de oliva español ha subido en casi 10 puntos porcentuales. Es de destacar el crecimiento que se ha producido en las importaciones a granel, de forma que una $\triangleright$ 
parte importante de la actividad embotelladora se está produciendo directamente en el país. En este formato a granel España es el primer exportador. Y, en total, Estados Unidos fue en 2014 el segundo destino del aceite de oliva español, tras las exportaciones con destino a Italia.

Por su parte, México es uno de los diez socios estratégicos de la Unión Europea en el mundo y el único que cuenta con un acuerdo global, tras el Acuerdo de Asociación Económica, Concertación Política y Cooperación, en vigor desde 2000, y la Asociación Estratégica acordada en 2008. Además, en julio de 2009, el aceite de oliva quedó libre de arancel y se eliminó el sistema de cuotas compensatorias. Estos hechos han favorecido que las importaciones mexicanas de aceite de oliva no hayan dejado de crecer desde el año 2000, habiéndose multiplicado por 3 en los últimos 15 años y por 10 en los últimos 25 años. A pesar de esta tendencia positiva, el consumo per cápita es aún muy escaso: en torno al $0,1 \mathrm{~kg} /$ habitante. $A$ diferencia de lo que sucede en los otros dos países seleccionados, España es el principal proveedor de aceite de oliva en México, con casi un $80 \%$ de la cuota de mercado. Sin embargo, para España, México es solo el 16 país de destino de las exportaciones.

Para la recogida de los datos se llevó a cabo durante 2015 una encuesta dirigida al censo de importadores alemanes, estadounidenses y mexicanos del proyecto «Alimentos de Extremadura - Foods from Spain». De las $37 \mathrm{em}$ presas que formaban el censo en 2014 se obtuvo respuesta de 31 de ellas: el $41,43 \%$ de los importadores alemanes, el $78,57 \%$ de los norteamericanos y el $100 \%$ de los mexicanos. El cuestionario se envió online a través de correo electrónico a los importadores, en español, inglés y alemán. Con la mayoría de los encuestados se contactó a través de la dirección de correo electrónico que se disponía, y en otros casos el contacto se realizó personalmente en la Feria World Olive Oil Exhibition.

Atendiendo al origen de los encuestados, la distribución de cuestionarios ha sido bastante homogénea, recibiéndose 10 cuestionarios de Alemania y México y 11 de Estados Unidos. Por tipo de canal, el 55\% de los importadores analizados trabajan preferentemente con establecimientos gourmet. El $46 \%$ de los encuestados comercializan mayoritariamente aceite español, mientras que el resto tiene una cartera equilibrada con aceites de los tres países.

Para valorar la imagen país productor se utilizaron como referencia los trabajos de Van Ittersum et al. (2003) y García-Gallego et al. (2015). Se creó una escala de 11 ítems que incluía distintos aspectos relacionados con la capacidad del país para producir aceitunas y aceite de oliva (Tabla 2). Los encuestados debían valorar dichos ítems para el caso de España, Italia y Grecia, utilizando para ello una escala de Likert de 7 puntos.

Para medir la preferencia del aceite de oliva español frente al de origen italiano y griego, los importadores debieron valorar del 1 al 7 su intención de compra en distintas situaciones: cuando las condiciones de compra del aceite de oliva español son similares respecto a las de los otros dos aceites e, incluso, cuando son peores (en términos de precio y condiciones de financiación).

\section{Resultados y discusión}

Los resultados generales sobre la imagen como país productor de aceite de oliva han mostrado que el país mejor valorado por el conjunto de importadores encuestados ha sido España $(6,11$ sobre 7$)$, y después Italia $(5,82) \triangleright$ 
TABLA 2

IMAGEN PAÍS-PRODUCTOR DE ESPAÑA, ITALIA Y GRECIA

\begin{tabular}{|c|c|c|c|}
\hline & España & Italia & Grecia \\
\hline Cuenta con personal cualificado en la producción y elaboración de aceite de oliva* & 6,26 & 6,00 & 5,06 \\
\hline Cuenta con una amplia experiencia de producción de aceite de oliva* & 6,35 & 6,16 & 5,55 \\
\hline Dispone del clima idóneo para la producción de aceitunas* & 6,65 & 6,13 & 5,97 \\
\hline El suelo y las tierras son adecuadas para la producción de aceitunas* & 6,71 & 6,16 & 5,90 \\
\hline En las producciones e industrias de aceite de oliva se garantiza adecuadamente la seguridad alimentaria* & 5,97 & 5,45 & 4,71 \\
\hline Las empresas agroalimentarias gozan de un sistema de trazabilidad completo* & 5,71 & 5,29 & 4,58 \\
\hline La calidad de las variedades de aceituna es muy buena* & 6,45 & 5,58 & 5,39 \\
\hline La experiencia del sector olivarero garantiza productos de alta calidad & 5,84 & 5,42 & 5,10 \\
\hline Los diseños de los aceites de oliva son variados y atractivos* & 5,52 & 6,32 & 4,65 \\
\hline Las empresas tienen capacidad para garantizar el suministro que se le solicite* & 5,94 & 5,55 & 4,55 \\
\hline $\begin{array}{l}\text { Las empresas ofrecen una oferta suficientemente amplia para atender a los distintos segmentos de } \\
\text { consumidores* }\end{array}$ & 5,87 & 5,97 & 4,65 \\
\hline Total & 6,11 & 5,82 & 5,10 \\
\hline
\end{tabular}

y Grecia $(5,10)$. En la Tabla 2 se puede observar que, en casi todos los ítems utilizados para medir la calidad del país como productor de aceite de oliva, España ha superado a los otros dos países mediterráneos. Tras realizar un análisis ANOVA de diferencia de medias entre las opiniones de los encuestados con respecto a cada país, las diferencias de valoración son estadísticamente significativas en 10 de los 11 ítems. Las diferencias más destacadas y, por tanto, los puntos fuertes de la imagen de España tienen que ver con las condiciones naturales: el clima, el suelo y la calidad de las variedades de aceitunas. Sin embargo, Italia ha superado a España en algunos ítems referidos a aspectos empresariales, como son la amplitud de la oferta y el atractivo y variedad de los diseños.

Tras medir la imagen país-productor para el conjunto de la muestra se ha analizado la imagen de España teniendo en cuenta el país de origen del importador. En los resultados se observa que, en general, Alemania tiene una percepción más baja de nuestro país en casi todos los ítems. Sin embargo, las diferencias entre las tres nacionalidades de importadores solo son significativas al $95 \%$ en las variables relativas a la garantía de la capacidad de suministro y a la capacidad de ofrecer un surtido de productos lo suficientemente amplio como para atender a la demanda de distintos perfiles de consumidores (Tabla 3). Tal y como puede observarse, los importadores de origen alemán valoran peor estas dos características que los importadores mexicanos y, sobre todo, los importadores norteamericanos.

En comparación con la imagen que tienen de los otros dos grandes países productores (Gráfico 1), los importadores de Alemania tienen una mejor valoración global de Italia como país productor que de España. Esto puede justificar el hecho de que la cuota de mercado del aceite español en dicho mercado sea más baja que la cuota del aceite italiano². En los tres mercados, Grecia es la que obtiene una valoración más baja de su imagen como productor.

De forma similar a lo anterior se ha realizado un análisis en función del tipo de $\triangleright$

2 Aunque el sector es consciente de que parte de la producción de aceite español se exporta a granel a Italia para ser comercializado bajo una marca del país transalpino. 
TABLA 3

IMAGEN DE ESPAÑA COMO PAÍS PRODUCTOR, SEGÚN EL ORIGEN DEL IMPORTADOR

\begin{tabular}{|c|c|c|c|c|}
\hline & Alemania & México & EEUU & Total \\
\hline Cuenta con personal cualificado en la producción y elaboración de aceite de oliva & 5,70 & 6,40 & 6,64 & 6,26 \\
\hline Cuenta con una amplia experiencia de producción de aceite de oliva & 6,00 & 6,50 & 6,55 & 6,35 \\
\hline Dispone del clima idóneo para la producción de aceitunas & 6,70 & 6,70 & 6,55 & 6,65 \\
\hline El suelo y las tierras son adecuadas para la producción de aceitunas & 6,80 & 6,70 & 6,64 & 6,71 \\
\hline $\begin{array}{l}\text { En las producciones e industrias de aceite de oliva se garantiza adecuadamente la } \\
\text { seguridad alimentaria }\end{array}$ & 5,90 & 5,80 & 6,18 & 5,97 \\
\hline Las empresas agroalimentarias gozan de un sistema de trazabilidad completo & 6,00 & 5,20 & 5,91 & 5,71 \\
\hline La calidad de las variedades de aceituna es muy buena & 6,10 & 6,60 & 6,64 & 6,45 \\
\hline La experiencia del sector olivarero garantiza productos de alta calidad & 5,60 & 5,60 & 6,27 & 5,84 \\
\hline Los diseños de los aceites de oliva son variados y atractivos & 5,10 & 5,40 & 6,00 & 5,52 \\
\hline Las empresas tienen capacidad para garantizar el suministro que se le solicite* & 5,20 & 6,00 & 6,55 & 5,94 \\
\hline $\begin{array}{l}\text { Las empresas ofrecen un surtido suficientemente amplio para atender a los distintos } \\
\text { segmentos de consumidores }{ }^{*}\end{array}$ & 5,00 & 5,90 & 6,64 & 5,87 \\
\hline Total & 5,83 & 6,07 & 6,41 & 6,11 \\
\hline
\end{tabular}

establecimiento para el que trabaja el importador. Según los resultados obtenidos, no existen diferencias estadísticamente significativas en las valoraciones de la imagen país-productor entre importadores que trabajan preferentemente con canales gourmet y los que trabajan con canales convencionales.

Por otro lado, se ha estudiado la intención de compra de aceite de oliva español, respecto a griego o italiano. En general, los resultados

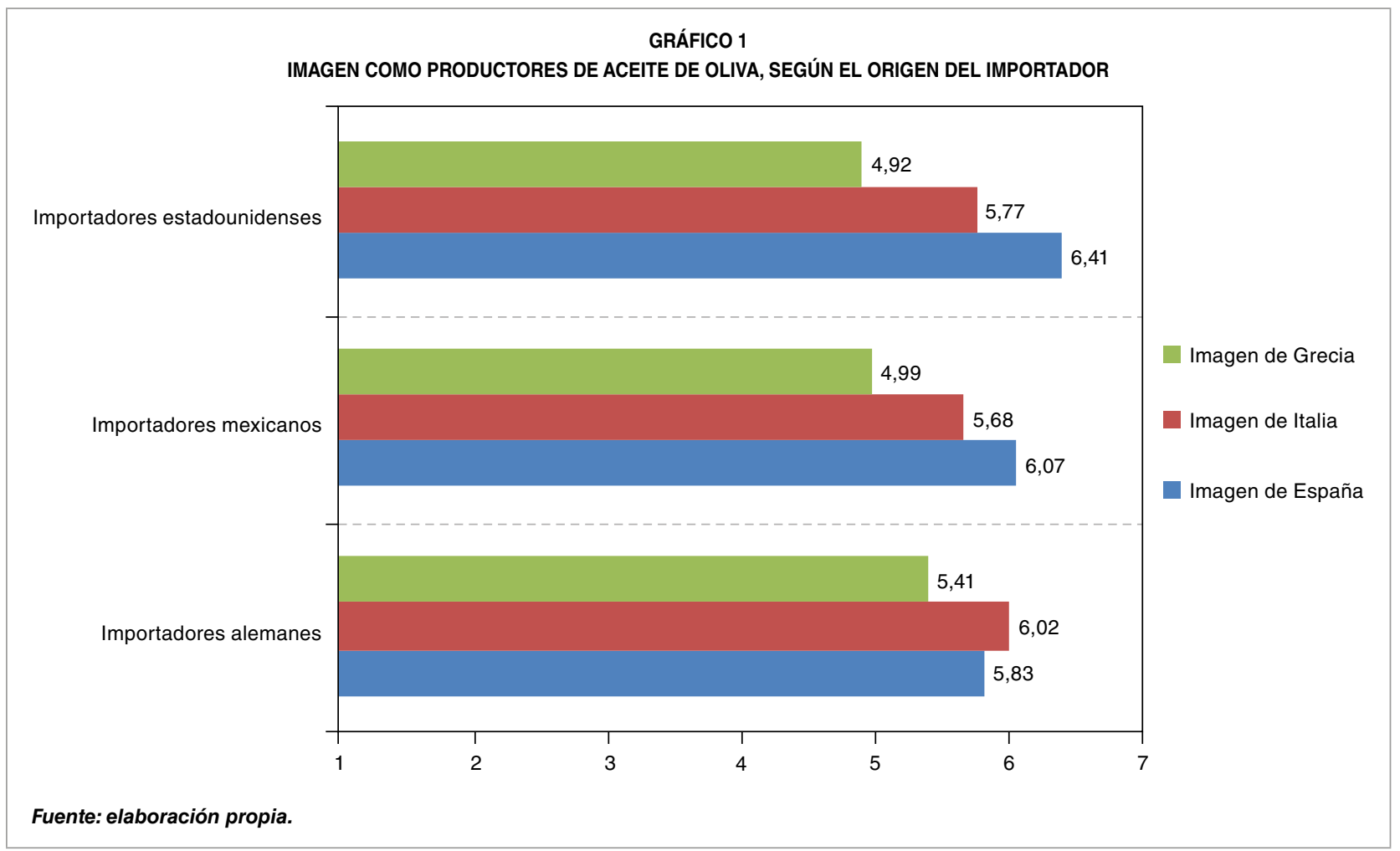


han puesto de manifiesto que los importadores opinan que siempre tendrán aceite de oliva español en su cartera de productos, y que, en igualdad de condiciones, preferirán un aceite de oliva español sobre un aceite de oliva griego y, en menor medida, sobre un aceite de oliva italiano (Tabla 4). Sin embargo, tal y como se podía esperar, ante peores condiciones del aceite de oliva español respecto a los otros aceites, el interés respecto a este disminuye, sobre todo cuando se compara con el de origen italiano.

La intención de adquirir preferentemente aceite de oliva español en distintas condiciones son similares para los importadores de los tres países analizados: Alemania, Estados Unidos y México. Tan solo se han detectado diferencias estadísticamente significativas respecto a la afirmación «Si tengo que elegir entre aceite de oliva español y de Italia, preferiré el español en igualdad de condiciones de compra», siendo México el país que más de acuerdo ha demostrado estar con esta afirmación, y después Estados Unidos y muy por detrás Alemania.

Además, se han comparado los resultados de las intenciones de compra entre los segmentos gourmet y no gourmet, pero no se han observado diferencias estadísticamente significativas entre ambos grupos.

\section{Conclusiones}

Diversos estudios han puesto de manifiesto la importancia del origen como criterio de compra de los consumidores de aceite de oliva en los mercados de países no productores, aunque solo en algunos de ellos se ha evaluado la preferencia entre distintos países de origen. Los resultados de los mismos reflejaban una preferencia mayor por el origen italiano que por otros orígenes, incluido en algunos casos el español (Mtimet et al., 2008; Menapace et al., 2011). Sin embargo, hasta donde ha llegado nuestra revisión de la literatura, estos estudios no han profundizado en las causas de esta preferencia evaluando la imagen de los países como productores de aceite de oliva.

Como indicaron Roth y Romeo (1992), evaluar y comprender cuándo la imagen del país de origen es positiva es una información relevante para los productores, ya que la pueden utilizar para resaltar $u$ omitir el país de origen en sus campañas de marketing. Además, puede ser utilizada por los Gobiernos para el diseño de sus campañas de promoción en mercados exteriores.

Con los resultados obtenidos en este estudio se puede concluir en primer lugar que los importadores consultados tienen una muy $\triangleright$

TABLA 4

INTENCIÓN DE COMPRA DE ACEITE ESPAÑOL

\begin{tabular}{|c|c|c|c|c|}
\hline & Media & Alemania & México & EEUU \\
\hline Siempre habrá aceite de oliva español en mis establecimientos & 6,06 & 5,60 & 6,20 & 6,36 \\
\hline $\begin{array}{l}\text { Si tengo que elegir entre aceite de oliva español e italiano, preferiré el español } \\
\text { en igualdad de condiciones de compra* }\end{array}$ & 4,77 & 3,50 & 5,90 & 4,91 \\
\hline $\begin{array}{l}\text { Si tengo que elegir entre aceite de oliva español e italiano, preferiré el español, } \\
\text { aunque las condiciones de compra sean algo peores }\end{array}$ & 3,81 & 3,00 & 4,40 & 4,00 \\
\hline $\begin{array}{l}\text { Si tengo que elegir entre aceite de oliva español y griego, preferiré el español } \\
\text { en igualdad de condiciones de compra }\end{array}$ & 5,16 & 4,40 & 6,30 & 4,82 \\
\hline $\begin{array}{l}\text { Si tengo que elegir entre aceite de oliva español y griego, preferiré el español, } \\
\text { aunque las condiciones de compra sean algo peores }\end{array}$ & 4,26 & 3,50 & 5,30 & 4,00 \\
\hline
\end{tabular}


buena imagen de España como productor de aceite de oliva en los tres mercados internacionales analizados. Esta buena imagen puede ser una de las causas que ha permitido que en los últimos años la cuota de mercado del aceite de oliva español se haya acercado considerablemente a la cuota de los aceites italianos tanto en el mercado estadounidense como en el alemán. Se puede considerar que la marca colectiva Foods from Spain constituye un adecuado reclamo en las campañas de promoción exterior del aceite de oliva nacional y es adecuado incluirla en el etiquetado de los envases del aceite.

Como segunda conclusión se extrae que, en términos de imagen, es el aceite italiano el principal competidor del aceite español, muy por encima del griego. En los mercados estadounidense y mexicano el aceite de oliva español está muy bien posicionado frente a los otros dos competidores internacionales, pero no así en el mercado alemán, donde la imagen de Italia como país de origen del aceite de oliva es mejor a la de España.

Los puntos débiles de la imagen de España tienen que ver más con aspectos comerciales y de marketing que con los procesos productivos propiamente dichos. Las peores valoraciones se obtienen en lo referido a la variedad de la oferta y el atractivo de sus diseños, precisamente los puntos fuertes de la oferta italiana. Es aquí, por tanto, donde el sector tiene que centrar sus esfuerzos en el futuro.

Finalmente, hay que indicar que esta investigación tiene ciertas limitaciones que hay que tener presente para interpretar correctamente los resultados. En primer lugar, el hecho de que la muestra analizada haya estado formada por los colaboradores del proyecto Alimentos de Extremadura del Gobierno regional significa que son importadores que ya están comprando producto español y que ya conocen España; es decir, su nivel de familiaridad con el origen es muy alto en todos los casos. Este hecho puede haber sesgado positivamente la valoración concedida al origen español frente al de los otros dos países competidores. Por ello, los resultados no deben ser extrapolables a otros importadores potenciales de aceite de oliva que aún no estén comprando producto español.

Por otro lado, el efecto lugar de origen no es universal y homogéneo para todos los ámbitos geográficos (Ahmed y D’Astous, 2007), por lo que los resultados de este estudio tampoco pueden extrapolarse a otros mercados diferentes a los tres analizados: Alemania, Estados Unidos y México. Igual que la preferencia por el aceite español y su imagen son mayores en México por la cercanía cultural, en otros mercados más próximos geográfica y culturalmente a Italia y Grecia, el aceite español puede no ser la primera opción de preferencia y su imagen puede no ser tan buena con respecto a otros países productores.

\section{Bibliografía}

[1] Ahmed, S. A., y d'Astous, A. (2007). Moderating effect of nationality on country-of-origin perceptions: English-speaking Thailand versus French-speaking Canada. Journal of Business Research, 60(3), 240-248.

[2] Askegaard, S., y Ger, G. (1998). Productcountry images: towards a contextualized approach. European Advances in Consumer Research, 3, 50-58.

[3] Bernabéu. R., Olmeda, M., Díaz, M., y Olivas, R. (2009). Oportunidades comerciales para el aceite de oliva de Castilla-La Mancha. Grasas y Aceites, 60(5), 525-533.

[4] Bilkey. W., y Nes, E. (1982). Country of origin effects on product evaluations. Journal of International Business Studies, 13(1), 89-99.

[5] Caporale, G., Policastro, S., Carlucci, A., y Monteleone, E. (2005). Consumer expectations $D$ 
for sensory properties in virgin olive oils. Food Quality and Preference, 17(1-2), 116-125.

[6] Chan-Halbrendt, C., Zhllima, E., Sisior, G., Imami, D., y Leonetti, L. (2010). Consumer Preferences for Olive Oil in Tirana, Albania. International Food and Agribusiness Management Review, 13(3), 55-74.

[7] COI (2019). Estadísticas de producción y exportación de aceite de oliva. Consejo Oleícola Internacional. Recuperado de www.internationaloliveoil.org

[8] Dekhili, S., y d'Hauteville, F. (2009). Effect of the region of origin on the perceived quality of olive oil: An experimental approach using a control group. Food Quality and Preference, 20(7), 525-532.

[9] Dekhili, S., Sirieix, L., y Cohen, E. (2011). How consumers choose olive oil: The importance of origin cues. Food Quality and Preference, 22(8), 757-762.

[10] Erraach, Y., Sayadi, S., Gómez, A. C., y ParraLópez, C. (2014). Consumer-stated preferences towards Protected Designation of Origin (PDO) labels in a traditional olive-oil-producing country: the case of Spain. New Medit, 13(4), 11-19.

[11] Espejel, J., y Fandos, C. (2008). La calidad percibida como antecedente de la intención de compra de aceite de oliva del Bajo Aragón con denominación de origen protegida. Esic Market (131), 231-275.

[12] Fotopoulos, C., y Krystallis, A. (2001). Are quality labels a real marketing advantage? A conjoint application on Greek PDO protected olive oil. Journal of International Food \& Agribusiness Marketing, 12(1), 1-22.

[13] García, M., Aragonés, Z., y Poole, N. (2002). A Repositioning Strategy for Olive Oil in the UK Market. Agribusiness, 18(2), 163-180.

[14] García-Gallego, J. M., Chamorro-Mera, A., y García-Galán, M. M. (2015). The region-of-origin effect in the purchase of wine: The moderating role of familiarity. Spanish Journal of Agricultural Research, 13(03).

[15] Han, C. M. (1989). Country of origin: Halo or summary construct? Journal of Marketing Research, 26(2), 222-229.

[16] Krystallis, A., y Ness, M. (2005). Consumer preferences for quality foods from a South European perspective: A conjoint analysis implementation on Greek olive oil. International
Food and Agribusiness Management Review, 8(2), 62-91.

[17] Menapace, L., Colson, G., Grebitus, C., y Facendola, M. (2011). Consumers' preferences for geographical origin labels: evidence from the Canadian olive oil market. European Review of Agricultural Economics, 38(2), 193-212.

[18] Molina, G. (2004). Sí Logo. Marca País. Marketing Global. Editorial Norma. España.

[19] Mtimet, N., Kashiwagi, A. K., Zaibet, L., y Masakazu, N. (2008). Exploring Japanese olive oil consumer behavior. 12th EAAE Congress 'People, Food and Environments: Global Trends and European Strategies'. Gent (Belgium), 26-29 August 2008.

[20] Mtimet, N., Zaibet, L., Zairi, C., y Hamida, H. (2013). Marketing Olive Oil Products in the Tunisian Local Market: The Importance of Quality Attributes and Consumers' Behavior. Journal of International Food \& Agribusiness Marketing, 25(2), 134-145.

[21] Panico, T., Del Giudice, T., y Caracciolo, F. (2014). Quality dimensions and consumer preferences: a choice experiment in the Italian extra virgin olive oil market. Agricultural Economics Review, 15(2), 100-112.

[22] Papadopoulos, N., y Heslop, L. A. (2003). Country equity and product-country images: state of art in research and implications. En Jain, S.C. (ed.), Handbook of Research in International Marketing, Edward Elgar, Cheltenham, 402-433.

[23] Roth, K., y Dimantopoulos, A. (2009). Advancing the country image construct. Journal of Business Research, 62(7), 726-740.

[24] Roth, M. S., y Romeo, J. B. (1992). Matching Product Category and Country Image Perceptions: A Framework for Managing Countryof-Origin Effects. Journal of International Business Studies, 23(3), 477-497.

[25] Scarpa, R., y Del Giudice, T. (2004). Market segmentation via mixed logit: extra-virgin olive oil in urban Italy. Journal of Agricultural \& Food Industrial Organization, 2(1).

[26] Van der Lans, I. A., Van Ittersum, K., De Cicco, A., y Loseby, M. (2001). The role of origin in consumer evaluation of food products. European Review of Agricultural Economics, 28(4), 451-477. 
[27] Van Ittersum, K., Candel, M., y Meulenberg, M. (2003). The influence of the image of a product's region of origin on product evaIuation. Journal of Business Research, 56(3), 215-226.

[28] Wang, C. L., Li, D., Barnes, B. R., y Ahn, J. (2012). Country image, product image and consumer purchase intention: Evidence from an emerging economy. International Business Review, 21(6), 1041-1051.
[29] Ward, R., Briz, J., y de Felipe, I. (2003). Competing Supplies of Olive Oil in the German Market: An Application of Multinomial Logit Models. Agribusiness, 19(3), 393-406.

[30] Yangui, A., Costa-Font, M., y Gil, J. M. (2014). Revealing additional preference heterogeneity with an extended random parameter logit model: the case of extra virgin olive oil. Spanish Journal of Agricultural Research, 12(3), 553-567. 